\title{
Incorporating Embedded Systems Security Awareness into a Computer Science Course via Minimal Interventions
}

\author{
Harini Ramaprasad, Julio César Bahamón, \\ Riley H. Jones \\ UNC Charlotte \\ \{hramapra,jbahamon,rjone159\}@uncc.edu
}

\author{
Stacey Watson \\ Rochester Institute of Technology \\ stacey.l.watson@rit.edu
}

\begin{abstract}
In this poster, we describe our research on the use of small units of content, termed minimal interventions, to create awareness of embedded systems security concepts in an undergraduate course, Introduction to Operating Systems and Networking. This work focuses on the incorporation of simple activities such as short readings, and the evaluation of their potential as teaching tools. To study the effectiveness of this minimal intervention approach, we collected student performance data over six (6) semesters and across multiple modalities of the course, with a total of 1,168 study participants. Students were asked to complete a short survey before and after completing the activities, to assess prior knowledge of the subject and measure knowledge gains that resulted from participation in the activity.
\end{abstract}

\section{CCS CONCEPTS}

- Social and professional topics $\rightarrow$ Computer science education; Student assessment.

\section{KEYWORDS}

Computer Science education, Embedded systems security

\section{INTRODUCTION}

The Internet is rapidly evolving to be an Internet of Things (IoT), in which a majority of connected artifacts are not traditional devices (such as PCs), but embedded devices. This motivates the need to increase awareness of embedded systems security among Computer Science (CS) students. Research suggests that integrating securityrelated concepts into multiple CS course curricula is more effective than just offering a single upper-level undergraduate computer security course. A study observed that by the end of a course that introduced CS through the lens of cybersecurity, students not only improved their awareness of security topics but also changed their behaviors as a result [1]. Another study implemented multiple security modules (e.g., integer/buffer overflow) into CS0 and CS1 courses and observed an increase in security knowledge based on a comparison of pre- and post-tests [2].

Permission to make digital or hard copies of part or all of this work for personal or classroom use is granted without fee provided that copies are not made or distributed for profit or commercial advantage and that copies bear this notice and the full citation on the first page. Copyrights for third-party components of this work must be honored. For all other uses, contact the owner/author(s).

SIGCSE '20, March 11-14, 2020, Portland, OR, USA

(C) 2020 Copyright held by the owner/author(s).

ACM ISBN 978-1-4503-6793-6/20/03.

https://doi.org/10.1145/3328778.3372666
While covering security-related topics in depth may be out of the scope of an Introduction to Operating Systems (OS) and Networking course, our research aims to discover whether small, targeted activities can improve students' general knowledge of these subjects without significantly increasing the didactic materials covered and also minimizing the students' workload.

\section{METHOD}

We created five short readings in our Intro to OS and Networking course. The readings introduced embedded systems security concepts related to the OS/networking topics of processes, CPU scheduling, memory management, file management and routers. Each reading was associated with a pre- and post-survey that established students' prior knowledge on the topic and measured any improvement in their knowledge, respectively. The readings and corresponding surveys were included in existing course modules, along with other components such as videos, exercises, and quizzes.

The readings and surveys were designed to require minimal effort to complete and to have no effect on student grades. We refer to these types of activities as minimal interventions. An example of a minimal intervention is a two-paragraph reading that introduces security considerations in memory management for embedded systems and a corresponding pair of pre- and post-surveys containing three questions about the readings' contents.

\section{CONTRIBUTIONS AND RESULTS}

This work implements and studies the effectiveness of minimal interventions to improve awareness of embedded systems security among undergraduate Computer Science students. With the proliferation of networked, embedded systems, such awareness is increasingly important. Preliminary results indicate that $20-32 \%$ of students gained knowledge as a result of these minimal interventions, even when there was no grade associated with the activities (which may result in some students submitting the surveys with no answers and others randomly choosing answers). In future work, we will evaluate whether having a small grade component associated with these minimal interventions produces more improvement.

\section{ACKNOWLEDGMENTS}

Our work was supported in part by NSF SaTC grant No. 1623268.

\section{REFERENCES}

[1] Tanya R. Flushman, Mark Gondree, and Zachary N. J. Peterson. 2015. This is Not a Game: Early Observations on Using Alternate Reality Games for Teaching Security Concepts to First-year Undergraduates. In Proceedings of CSET'15. USENIX Association, 1. http://dl.acm.org/citation.cfm?id=2831120.2831121

[2] Blair Taylor and Shiva Azadegan. 2008. Moving Beyond Security Tracks: Integrating Security in CS0 and CS1. In SIGCSE '08. ACM, 320-324. https://doi.org/10. $1145 / 1352135.1352246$ 